\title{
OBSERVER-BASED CONTROLLER DESIGN OF TIME-DELAY SYSTEMS WITH AN INTERVAL TIME-VARYING DELAY
}

\author{
Mai Viet THUAN * , Vu Ngoc PHAT **, HiEU TRINH *** \\ * Department of Mathematics and Informatics \\ Thai Nguyen University of Sciences, Quyet Thang Ward, Thai Nguyen City 23000, Vietnam \\ e-mail: maithuank1@gmail.com \\ ** Institute of Mathematics \\ VAST, 18 Hoang Quoc Viet Road, Hanoi 10307, Vietnam \\ e-mail: vnphat@math.ac.vn \\ ${ }^{* * *}$ School of Engineering \\ Deakin University, Geelong, VIC 3217, Australia \\ e-mail: hieu.trinh@deakin.edu.au
}

\begin{abstract}
This paper considers the problem of designing an observer-based output feedback controller to exponentially stabilize a class of linear systems with an interval time-varying delay in the state vector. The delay is assumed to vary within an interval with known lower and upper bounds. The time-varying delay is not required to be differentiable, nor should its lower bound be zero. By constructing a set of Lyapunov-Krasovskii functionals and utilizing the Newton-Leibniz formula, a delay-dependent stabilizability condition which is expressed in terms of Linear Matrix Inequalities (LMIs) is derived to ensure the closed-loop system is exponentially stable with a prescribed $\alpha$-convergence rate. The design of an observerbased output feedback controller can be carried out in a systematic and computationally efficient manner via the use of an LMI-based algorithm. A numerical example is given to illustrate the design procedure.
\end{abstract}

Keywords: observer-based feedback control, interval time-varying delay, linear matrix inequalities, Lyapunov-Krasovskii functionals, exponential stability.

\section{Introduction}

Time-delay systems have attracted widespread attention, and many fundamental problems including stability, stabilization and estimation have been reported and studied in the literature (see, for example, the works of Blizorukova et al. (2001), Busłowicz (2010), Fridman and Shaked (2002), Gu et al. (2003), Kaczorek and Busłowicz (2004), Kowalewski (2009), Park (1999), Raja et al. (2011), Richard (2003), Tokarzewski (2009), Trinh (1994; 1997; 1999; 2010), Xiang et al. (2010) and the references therein). In particular, a problem of theoretical and practical importance is the design of observer-based controllers or dynamic output feedback controllers for time-delay systems. This problem stems from a practical constraint that not all of the state variables are available for feedback control, and therefore either an observer-based or a dynamical output feedback controller, which uses only the available output information, is employed to achieve the desired closed-loop system performance. In this regard, the Lyapunov-Krasovskii functional approach, which resulted in Riccati equations, LMIs or matrix inequalities, has been among some popular and effective tools used in the design of observer-based and dynamic output feedback controllers for time-delay systems (Baser and Kizilsac, 2007; Chen, 2007; Ivanescu et al., 2000; Kwon et al., 2006; Park, 2004; Tong et al., 2011). So far, the existing works have treated the time delay as either constant (Chen, 2007; Ivanescu et al., 2000; Kwon et al., 2006; Park, 2004) or time-varying (Baser and Kizilsac, 2007), but with the assumption that the time-varying delay is a continuously differentiable function satisfying some boundedness conditions on its derivative.

There has been growing research interest in stability analysis and stabilization of systems subject to an interval 
time-varying delay (see, for example, Botmart et al., 2011; Phat et al., 2012; Shao, 2009; Shao and Han, 2012). An interval time-varying delay is a time delay that varies within an interval whose lower bound is not restricted to be zero and its time derivative can even be undefined or unknown. Recently, exponential stability of linear systems with an interval time-varying non-differentiable delay was considered by Phat et al. (2012). Also, the problem of designing memoryless state feedback controllers to exponentially stabilize a class of linear uncertain systems with an interval time-varying delay was studied by Botmart et al. (2011). When not all of the state variables are available for feedback control, a state observer may be used to provide an estimate of the state vector and thus an observer-based output feedback controller can be realized. Nevertheless, compared with the state feedback design problem (Botmart et al., 2011), the observer-based output feedback design problem involves more control parameters and it is more complicated and difficult to solve using a convex programming approach.

This paper specifically focuses on interval time-varying delays. We study a class of linear systems subject to an interval time-varying delay in the state vector. The time-varying delay varies within an interval with known lower and upper bounds. Here, the delay is not required to be differentiable nor should its lower bound be zero. Under the practical constraint that not all of the state variables of the system are available for feedback control, we consider the problem of designing an observer-based output feedback controller to exponentially stabilize the closed-loop system with a prescribed $\alpha$-convergence rate. Since the real-time knowledge of the delay is not available and completely unknown, it is clear that no proposed structure for the state observer can contain any internal delay. This is in contrast to the existing observer-based design methods (Chen, 2007; Ivanescu et al., 2000; Kwon et al., 2006; Park, 2004), which treated a known constant time delay and thus their proposed state observers containing an internal time delay.

In this paper, we propose to use a completely memoryless full-order Luenberger-type state observer to reconstruct the state vector for the feedback control purpose. Our objective is to systematically derive both the observer and controller gain matrices to ensure that the closed-loop system is exponentially stable. By constructing a set of Lyapunov-Krasovskii functionals and utilizing the Newton-Leibniz formula, a delay-dependent LMI stabilizability condition is derived. As a result, both the controller and observer parameters can be derived via the use of an efficient LMI-based algorithm.

Notation. The following notation will be used in this paper: $\mathbb{R}^{+}$denotes the set of all real non-negative numbers; $\mathbb{R}^{n}$ denotes the $n$-dimensional space with the vector norm $\|\cdot\| ; M^{n \times r}$ denotes the space of all $(n \times$ $r$ )-dimensional matrices; $A^{T}$ denotes the transpose of matrix $A ; A$ is symmetric if $A=A^{T} ; I$ denotes the identity matrix; $\lambda(A)$ denotes the set of all eigenvalues of $A ; \lambda_{\max }(A)=\max \{\operatorname{Re} \lambda ; \lambda \in \lambda(A)\}$;

$$
\begin{aligned}
x_{t}:=\{x(t+s): s & \in[-h, 0]\}, \\
& \left\|x_{t}\right\|=\sup _{s \in[-h, 0]}\|x(t+s)\|
\end{aligned}
$$

$\mathcal{C}^{1}\left([0, t], \mathbb{R}^{n}\right)$ denotes the set of all $\mathbb{R}^{n}$-valued continuously differentiable functions on $[0, t]$. A matrix $A$ is called semi-positive definite $(A \geq 0)$ if $\langle A x, x\rangle \geq 0$, for all $x \in \mathbb{R}^{n} ; A$ is positive definite $(A>0)$ if $\langle A x, x\rangle>0$ for all $x \neq 0 ; A>B$ means $A-B>0$. The symmetric term in a matrix is denoted by $*$.

\section{Problem statement and preliminaries}

Consider a linear system with an interval time-varying delay in the state vector, where

$$
\begin{aligned}
& \dot{x}(t)=A_{0} x(t)+A_{1} x(t-h(t))+B u(t), t \geq 0, \\
& y(t)=C x(t),
\end{aligned}
$$

with the initial condition function

$$
x(t)=\phi(t), \quad t \in\left[-h_{2}, 0\right],
$$

where $x(t) \in \mathbb{R}^{n}$ is the state vector, $u(t) \in \mathbb{R}^{m}$ is a control vector, $y(t) \in \mathbb{R}^{q}$ is the output vector, $A_{0}, A_{1} \in$ $\mathbb{R}^{n \times n}, B \in \mathbb{R}^{n \times m}$ and $C \in \mathbb{R}^{q \times n}$ are constant matrices, $\phi(t) \in \mathcal{C}^{1}\left(\left[-h_{2}, 0\right], \mathbb{R}^{n}\right)$ is the initial function with the norm

$$
\|\phi\|_{C^{1}}=\sup _{-h_{2} \leq t \leq 0}\{\|\phi(t)\|,\|\dot{\phi}(t)\|\} .
$$

The time-varying delay function $h(t)$ satisfies

$$
0<h_{1} \leq h(t) \leq h_{2}, \quad h_{1}<h_{2}, \quad t \geq 0 .
$$

It is worth noting that the time delay is assumed to be a continuous function belonging to a given interval, which means that the lower and upper bounds to the time-varying delay are available, but the lower bound is not restricted to being zero. Furthermore, the time-varying delay can be non-differentiable.

Since not all of the state variables are available for feedback control and the real-time knowledge of the delay, $h(t)$, is not available, we consider a completely memoryless full-order Luenberger state observer for the system (1),

$$
\begin{aligned}
& \dot{\hat{x}}(t)=A_{0} \hat{x}(t)+B u(t)+L(y(t)-C \hat{x}(t)), \quad t \geq 0, \\
& \hat{x}(t)=0, \quad \forall t \in\left[-h_{2}, 0\right],
\end{aligned}
$$


and the following control law for the system (1):

$$
u(t)=-K \hat{x}(t), \quad t \geq 0,
$$

in which $\hat{x}(t) \in \mathbb{R}^{n}$ is the observer state vector, $K \in$ $\mathbb{R}^{m \times n}$ and $L \in \mathbb{R}^{n \times q}$ are the constant controller and observer gain matrices, respectively.

Define an error vector $\Theta(t)=x(t)-\hat{x}(t)$, which denotes the difference between the actual state and estimated states. Then we have the following closed-loop system:

$$
\begin{aligned}
\dot{\Theta}(t)= & \left(A_{0}-L C\right) \Theta(t)+A_{1} x(t-h(t)), \\
\dot{x}(t)= & \left(A_{0}-B K\right) x(t)+A_{1} x(t-h(t)) \\
& +B K \Theta(t) .
\end{aligned}
$$

The objective is to derive $K$ and $L$ so that the above closed-loop system is exponentially stable with a prescribed $\alpha$-convergence rate.

Definition 1. (Phat et al., 2012) Given $\alpha>0$, the system (1), where $u(t)=0$, is $\alpha$-stable if there exists a positive number $\beta>0$ such that every solution $x(t, \phi)$ satisfies the condition

$$
\|x(t, \phi)\| \leq \beta e^{-\alpha t}\|\phi\|_{\mathcal{C}^{1}}, \quad \forall t \geq 0 .
$$

We also introduce the following technical well-known propositions, which will be used in the proof of our results.

Proposition 1. (Jensen's inequality (Gu et al., 2003)) For any symmetric positive definite matrix $M>0$, a scalar $\gamma>0$ and a vector function $\omega:[0, \gamma] \rightarrow \mathbb{R}^{n}$ such that the integrations concerned are well defined, the following inequality holds:

$$
\begin{array}{r}
\left(\int_{0}^{\gamma} \omega(s) \mathrm{d} s\right)^{T} M\left(\int_{0}^{\gamma} \omega(s) \mathrm{d} s\right) \\
\leq \gamma\left(\int_{0}^{\gamma} \omega^{T}(s) M \omega(s) \mathrm{d} s\right) .
\end{array}
$$

Proposition 2. (Schur complement lemma) Given constant matrices $X, Y, Z$ with appropriate dimensions satisfying $X=X^{T}, Y=Y^{T}$ and $Y>0$, we have $X+Z^{T} Y^{-1} Z<0$ if and only if

$$
\left(\begin{array}{cc}
X & Z^{T} \\
Z & -Y
\end{array}\right)<0 \quad \text { or } \quad\left(\begin{array}{cc}
-Y & Z \\
Z^{T} & X
\end{array}\right)<0
$$

\section{Main results}

In this section, using the Lyapunov-Krasovskii method, we establish a delay-dependent criterion in terms of linear matrix inequalities for the exponential stabilization of the linear system (1) via the observer-based controller (3)-(4).
Now, we synthesize the gain matrices $K$ and $L$ of controller and observer as follows:

$$
K=Y P^{-1}, \quad L=-\frac{1}{2} P C^{T},
$$

where $P$ is a positive-definite matrix and $Y$ are to be designed.

Write

$h_{12}=h_{2}-h_{1}$,

$\mathcal{H}=\left[\begin{array}{lllllll}M_{1}^{T} & M_{2}^{T} & 0 & 0 & 0 & 0 & 0\end{array}\right]^{T}$,

$\mathcal{L}=\left[\begin{array}{lllllll}N_{1}^{T} & N_{2}^{T} & 0 & 0 & 0 & 0 & 0\end{array}\right]^{T}$,

$\Omega=\left[\begin{array}{ccccccc}\Omega_{11} & \Omega_{12} & \Omega_{13} & -N_{1} & \Omega_{15} & B Y & 0 \\ * & \Omega_{22} & M_{2} & -N_{2} & P A_{1}^{T} & P A_{1}^{T} & 0 \\ * & * & \Omega_{33} & 0 & 0 & 0 & 0 \\ * & * & * & \Omega_{44} & 0 & 0 & 0 \\ * & * & * & * & \Omega_{55} & B Y & 0 \\ * & * & * & * & * & \Omega_{66} & P C^{T} \\ * & * & * & * & * & * & -I\end{array}\right]$,

with

$$
\begin{aligned}
\Omega_{11}= & A_{0} P+P A_{0}^{T}+2 \alpha P-B Y-Y^{T} B^{T} \\
& +Q_{1}+Q_{2}-e^{-2 \alpha h_{1}} S_{1}, \\
\Omega_{12}= & N_{1}-M_{1}+A_{1} P, \quad \Omega_{13}=M_{1}+e^{-2 \alpha h_{1}} S_{1}, \\
\Omega_{15}= & P A_{0}^{T}-Y^{T} B^{T}, \\
\Omega_{22}= & N_{2}+N_{2}^{T}-M_{2}-M_{2}^{T}, \\
\Omega_{33}= & -e^{-2 \alpha h_{1}} Q_{1}-e^{-2 \alpha h_{1}} S_{1}, \\
\Omega_{44}= & -e^{-2 \alpha h_{2}} Q_{2}, \quad \Omega_{55}=h_{1}^{2} S_{1}+h_{12} S_{2}-2 P, \\
\Omega_{66}= & A_{0} P+P A_{0}^{T}+2 \alpha P, \\
\lambda= & \lambda_{\min }\left(P^{-1}\right), \\
\Lambda=2 & \lambda_{\max }\left(P^{-1}\right)+h_{1} \lambda_{\max }\left(P^{-1} Q_{1} P^{-1}\right) \\
& +h_{2} \lambda_{\max }\left(P^{-1} Q_{2} P^{-1}\right)+\frac{1}{2} h_{1}^{3} \lambda_{\max }\left(P^{-1} S_{1} P^{-1}\right) \\
& +\frac{1}{2}\left(h_{2}^{2}-h_{1}^{2}\right) \lambda_{\max }\left(P^{-1} S_{2} P^{-1}\right) .
\end{aligned}
$$

Theorem 1. Given $\alpha>0, u(t)=-Y P^{-1} \hat{x}(t)$ is an observer-based controller for the system (1) if there exist symmetric positive-definite matrices $P, S_{1}, S_{2}, Q_{1}, Q_{2}$, and matrices $M_{1}, M_{2}, N_{1}, N_{2}, Y$ satisfying the following LMIs:

$$
\begin{aligned}
& \Omega_{1}=\left[\begin{array}{cc}
\Omega & h_{12} \mathcal{H} \\
h_{12} \mathcal{H}^{T} & -h_{12} e^{-2 \alpha h_{2}} S_{2}
\end{array}\right]<0, \\
& \Omega_{2}=\left[\begin{array}{cc}
\Omega & h_{12} \mathcal{L} \\
h_{12} \mathcal{L}^{T} & -h_{12} e^{-2 \alpha h_{2}} S_{2}
\end{array}\right]<0 .
\end{aligned}
$$

Moreover, the solution $x(t, \phi)$ satisfies

$$
\|x(t, \phi)\| \leq \sqrt{\frac{\Lambda}{\lambda}} e^{-\alpha t}\|\phi\|_{\mathcal{C}^{1}}, \quad t \in \mathbb{R}^{+} .
$$


Proof. For $i=1,2$ set

$$
\begin{aligned}
\overline{Q_{i}} & =P^{-1} Q_{i} P^{-1}, & \overline{S_{i}} & =P^{-1} S_{i} P^{-1}, \\
\overline{M_{i}} & =P^{-1} M_{i} P^{-1}, & \overline{N_{i}} & =P^{-1} N_{i} P^{-1} .
\end{aligned}
$$

Consider the following Lyapunov-Krasovskii functional:

$$
V=\sum_{i=1}^{5} V_{i}
$$

where

$$
\begin{aligned}
& V_{1}=x^{T}(t) P^{-1} x(t)+\Theta^{T}(t) P^{-1} \Theta(t), \\
& V_{2}=\int_{t-h_{1}}^{t} e^{2 \alpha(s-t)} x^{T}(s) \overline{Q_{1}} x(s) \mathrm{d} s \\
& V_{3}=\int_{t-h_{2}}^{t} e^{2 \alpha(s-t)} x^{T}(s) \overline{Q_{2}} x(s) \mathrm{d} s, \\
& V_{4}=\int_{-h_{1}}^{0} \int_{t+s}^{t} h_{1} e^{2 \alpha(\tau-t)} \dot{x}^{T}(\tau) \overline{S_{1}} \dot{x}(\tau) \mathrm{d} \tau \mathrm{d} s, \\
& V_{5}=\int_{-h_{2}}^{-h_{1}} \int_{t+s}^{t} e^{2 \alpha(\tau-t)} \dot{x}^{T}(\tau) \overline{S_{2}} \dot{x}(\tau) \mathrm{d} \tau \mathrm{d} s .
\end{aligned}
$$

Taking the derivative of $V_{i}, i=1, \ldots, 5$, we have

$$
\begin{aligned}
\dot{V}_{1} & =x^{T}(t)\left[P^{-1}\left[A_{0}-B K\right]+\left[A_{0}-B K\right]^{T} P^{-1}\right] x(t) \\
& +2 x^{T}(t) P^{-1} A_{1} x(t-h(t))+2 x^{T}(t) P^{-1} B K \Theta(t) \\
& +\Theta^{T}(t)\left[P^{-1}\left[A_{0}-L C\right]+\left[A_{0}-L C\right]^{T} P^{-1}\right] \Theta(t) \\
& +2 \Theta^{T}(t) P^{-1} A_{1} x(t-h(t)),
\end{aligned}
$$

$$
\begin{aligned}
\dot{V}_{2}= & -2 \alpha V_{2}+x^{T}(t) \overline{Q_{1}} x(t) \\
& -e^{-2 \alpha h_{1}} x^{T}\left(t-h_{1}\right) \overline{Q_{1}} x\left(t-h_{1}\right), \\
\dot{V}_{3}= & -2 \alpha V_{3}+x^{T}(t) \overline{Q_{2}} x(t) \\
& -e^{-2 \alpha h_{2}} x^{T}\left(t-h_{2}\right) \overline{Q_{2}} x\left(t-h_{2}\right), \\
\dot{V}_{4}= & -2 \alpha V_{4}+h_{1}^{2} \dot{x}^{T}(t) \overline{S_{1}} \dot{x}(t) \\
& -e^{-2 \alpha h_{1}} \int_{t-h_{1}}^{t} h_{1} \dot{x}^{T}(s) \overline{S_{1}} \dot{x}(s) \mathrm{d} s,
\end{aligned}
$$$$
\dot{V}_{5} \leq-2 \alpha V_{5}+h_{12} \dot{x}^{T}(t) \overline{S_{2}} \dot{x}(t)
$$$$
-e^{-2 \alpha h_{2}} \int_{t-h_{2}}^{t-h_{1}} \dot{x}^{T}(s) \overline{S_{2}} \dot{x}(s) \mathrm{d} s .
$$

Using Proposition 1, we get

$$
\begin{aligned}
& -\int_{t-h_{1}}^{t} h_{1} \dot{x}^{T}(s) \overline{S_{1}} \dot{x}(s) \mathrm{d} s \\
& \quad \leq-\left(x(t)-x\left(t-h_{1}\right)\right)^{T} \overline{S_{1}}\left(x(t)-x\left(t-h_{1}\right)\right) .
\end{aligned}
$$

By the Leibniz-Newton formula, we have

$$
\begin{aligned}
-e^{-2 \alpha h_{2}} & \int_{t-h_{2}}^{t-h_{1}} \dot{x}^{T}(s) \overline{S_{2}} \dot{x}(s) \mathrm{d} s \\
= & -e^{-2 \alpha h_{2}} \int_{t-h_{2}}^{t-h(t)} \dot{x}^{T}(s) \overline{S_{2}} \dot{x}(s) \mathrm{d} s \\
& -e^{-2 \alpha h_{2}} \int_{t-h(t)}^{t-h_{1}} \dot{x}^{T}(s) \overline{S_{2}} \dot{x}(s) \mathrm{d} s \\
+ & 2 \zeta^{T}(t) \mathcal{M}\left[x\left(t-h_{1}\right)-x(t-h(t))\right. \\
& \left.-\int_{t-h(t)}^{t-h_{1}} \dot{x}(s) \mathrm{d} s\right] \\
+ & 2 \zeta^{T}(t) \mathcal{N}\left[x(t-h(t))-x\left(t-h_{2}\right)\right. \\
& \left.-\int_{t-h_{2}}^{t-h(t)} \dot{x}(s) \mathrm{d} s\right]
\end{aligned}
$$

where

$$
\begin{aligned}
& \mathcal{M}=\left[\begin{array}{llllll}
\bar{M}^{T} & {\overline{M_{2}}}^{T} & 0 & 0 & 0 & 0
\end{array}\right]^{T}, \\
& \mathcal{N}=\left[\begin{array}{llllll}
{\overline{N_{1}}}^{T} & {\overline{N_{2}}}^{T} & 0 & 0 & 0 & 0
\end{array}\right]^{T}, \\
& \zeta(t)=\left[\begin{array}{lll}
x^{T}(t) & x^{T}(t-h(t)) & x^{T}\left(t-h_{1}\right)
\end{array}\right. \\
& \left.x^{T}\left(t-h_{2}\right) \quad \dot{x}^{T}(t) \quad \Theta^{T}(t)\right]^{T} .
\end{aligned}
$$

From (6) it follows that

$$
\begin{gathered}
2 \dot{x}^{T}(t) P^{-1}\left[\left[A_{0}-B K\right] x(t)+A_{1} x(t-h(t))\right. \\
+B K \Theta(t)-\dot{x}(t)]=0 .
\end{gathered}
$$

From (8)-(14), we have

$$
\begin{aligned}
& \dot{V}+2 \alpha V \\
& \leq \zeta^{T}(t) \Xi \zeta(t) \\
& -\int_{t-h(t)}^{t-h_{1}}\left[2 \zeta^{T}(t) \mathcal{M} \dot{x}(s)+\dot{x}^{T}(s) e^{-2 \alpha h_{2}} \overline{S_{2}} \dot{x}(s)\right] \mathrm{d} s \\
& -\int_{t-h_{2}}^{t-h(t)}\left[2 \zeta^{T}(t) \mathcal{N} \dot{x}(s)+\dot{x}^{T}(s) e^{-2 \alpha h_{2}} \overline{S_{2}} \dot{x}(s)\right] \mathrm{d} s \\
& =h_{12}^{-1} \int_{t-h(t)}^{t-h_{1}}\left\{\zeta^{T}(t) \Xi \zeta(t)-2 h_{12} \zeta^{T}(t) \mathcal{M} \dot{x}(s)\right. \\
& \left.-h_{12} \dot{x}^{T}(s) e^{-2 \alpha h_{2}} \overline{S_{2}} \dot{x}(s)\right\} \mathrm{d} s \\
& +h_{12}^{-1} \int_{t-h_{2}}^{t-h(t)}\left\{\zeta^{T}(t) \Xi \zeta(t)-2 h_{12} \zeta^{T}(t) \mathcal{N} \dot{x}(s)\right. \\
& \left.-h_{12} \dot{x}^{T}(s) e^{-2 \alpha h_{2}} \overline{S_{2}} \dot{x}(s)\right\} \mathrm{d} s
\end{aligned}
$$




$$
\begin{aligned}
= & h_{12}^{-1} \int_{t-h(t)}^{t-h_{1}}\left[\begin{array}{l}
\zeta(t) \\
\dot{x}(s)
\end{array}\right]^{T}\left[\begin{array}{cc}
\Xi & -h_{12} \mathcal{M} \\
-h_{12} \mathcal{M}^{T} & -h_{12} e^{-2 \alpha h_{2}} \overline{S_{2}}
\end{array}\right] \\
& \times\left[\begin{array}{c}
\zeta(t) \\
\dot{x}(s)
\end{array}\right] \mathrm{d} s \\
& +h_{12}^{-1} \int_{t-h_{2}}^{t-h(t)}\left[\begin{array}{c}
\zeta(t) \\
\dot{x}(s)
\end{array}\right]^{T}\left[\begin{array}{cc}
\Xi & -h_{12} \mathcal{N} \\
-h_{12} \mathcal{N}^{T} & -h_{12} e^{-2 \alpha h_{2}} \overline{S_{2}}
\end{array}\right] \\
& \times\left[\begin{array}{c}
\zeta(t) \\
\dot{x}(s)
\end{array}\right] \mathrm{d} s,
\end{aligned}
$$

where

$$
\Xi=\left[\begin{array}{cccccc}
\Xi_{11} & \Xi_{12} & \Xi_{13} & -\overline{N_{1}} & \Xi_{15} & P^{-1} B K \\
* & \Xi_{22} & \overline{M_{2}} & -\overline{N_{2}} & A_{1}^{T} P^{-1} & A_{1}^{T} P^{-1} \\
* & * & \Xi_{33} & 0 & 0 & 0 \\
* & * & * & \Xi_{44} & 0 & 0 \\
* & * & * & * & \Xi_{55} & P^{-1} B K \\
* & * & * & * & * & \Xi_{66}
\end{array}\right]
$$

with

$$
\begin{aligned}
\Xi_{11}= & P^{-1}\left[A_{0}-B K\right]+\left[A_{0}-B K\right]^{T} P^{-1} \\
& +2 \alpha P^{-1}+\overline{Q_{1}}+\overline{Q_{2}}-e^{-2 \alpha h_{1}} \overline{S_{1}}, \\
\Xi_{12}= & \overline{N_{1}}-\overline{M_{1}}+P^{-1} A_{1}, \\
\Xi_{13}= & \overline{M_{1}}+e^{-2 \alpha h_{1}} \overline{S_{1}}, \\
\Xi_{15}= & A_{0}^{T} P^{-1}-K^{T} B^{T} P^{-1}, \\
\Xi_{22}= & \overline{N_{2}}+{\overline{N_{2}}}^{T}-\overline{M_{2}}-{\overline{M_{2}}}^{T}, \\
\Xi_{33}= & -e^{-2 \alpha h_{1}} \overline{Q_{1}}-e^{-2 \alpha h_{1}} \overline{S_{1}}, \\
\Xi_{44}= & -e^{-2 \alpha h_{2}} \overline{Q_{2}}, \\
\Xi_{55}= & h_{1}^{2} \overline{S_{1}}+h_{12} \overline{S_{2}}-2 P^{-1}, \\
\Xi_{66}= & P^{-1} A_{0}+A_{0}^{T} P^{-1}+2 \alpha P^{-1} \\
& -P^{-1} L C-C^{T} L^{T} P^{-1} .
\end{aligned}
$$

Setting

$$
\Upsilon_{1}=\left[\begin{array}{cc}
\Xi & -h_{12} \mathcal{M} \\
-h_{12} \mathcal{M}^{T} & -h_{12} e^{-2 \alpha h_{2}} \overline{S_{2}}
\end{array}\right]
$$

and

$$
\Upsilon_{2}=\left[\begin{array}{cc}
\Xi & -h_{12} \mathcal{N} \\
-h_{12} \mathcal{N}^{T} & -h_{12} e^{-2 \alpha h_{2}} \overline{S_{2}}
\end{array}\right]
$$

we have

$$
\begin{aligned}
\dot{V}\left(t, x_{t},\right. & \Theta(t))+2 \alpha V\left(t, x_{t}, \Theta(t)\right) \\
\leq & h_{12}^{-1} \int_{t-h(t)}^{t-h_{1}}\left[\begin{array}{c}
\zeta(t) \\
\dot{x}(s)
\end{array}\right]^{T} \Upsilon_{1}\left[\begin{array}{c}
\zeta(t) \\
\dot{x}(s)
\end{array}\right] \mathrm{d} s \\
& +h_{12}^{-1} \int_{t-h_{2}}^{t-h(t)}\left[\begin{array}{c}
\zeta(t) \\
\dot{x}(s)
\end{array}\right]^{T} \Upsilon_{2}\left[\begin{array}{c}
\zeta(t) \\
\dot{x}(s)
\end{array}\right] \mathrm{d} s .
\end{aligned}
$$

We have to demonstrate that $\Upsilon_{1}<0$ is equivalent to $\Omega_{1}<0$ and $\Upsilon_{2}<0$ is equivalent to $\Omega_{2}<0$. Indeed, we pre- and post-multiply both sides of $\Upsilon_{1}, \Upsilon_{2}$ with

$$
\operatorname{diag}\{P, P, P, P, P, P, P\}
$$

and, using the Schur complement lemma and the condition (7), we have that $\Upsilon_{1}<0$ is equivalent to $\Omega_{1}<0$ and $\Upsilon_{2}<0$ is equivalent to $\Omega_{2}<0$. Therefore,

$$
\dot{V}\left(t, x_{t}, \Theta(t)\right)+2 \alpha V\left(t, x_{t}, \Theta(t)\right) \leq 0, \quad \forall t \geq 0 .
$$

Integrating both the sides of (19) from 0 to $t$, we obtain

$$
V\left(t, x_{t}, \Theta(t)\right) \leq V\left(0, x_{0}, \Theta(0)\right) e^{-2 \alpha t}, \quad \forall t \in \mathbb{R}^{+} .
$$

By simple computation, we have

$$
\begin{aligned}
& V\left(t, x_{t}, \Theta(t)\right) \\
& \quad \geq \lambda_{\min }\left(P^{-1}\right)\|x(t)\|^{2}+\lambda_{\min }\left(P^{-1}\right)\|\Theta(t)\|^{2} \\
& \quad \geq \lambda_{\min }\left(P^{-1}\right)\|x(t)\|^{2}=\lambda\|x(t)\|^{2}, \quad \forall t \geq 0,
\end{aligned}
$$

and

$$
V\left(0, x_{0}, \Theta(0)\right) \leq \Lambda\|\phi\|_{\mathcal{C}^{1}}^{2} .
$$

Hence

$$
\begin{aligned}
& \lambda\|x(t, \phi)\|^{2} \leq V\left(t, x_{t}, \Theta(0)\right) \leq V\left(0, x_{0}, \Theta(0)\right) e^{-2 \alpha t} \\
& \leq \Lambda e^{-2 \alpha t}\|\phi\|_{\mathcal{C}^{1}}^{2},
\end{aligned}
$$

and the solution $x(t, \phi)$ of the system satisfies

$$
\|x(t, \phi)\| \leq \sqrt{\frac{\Lambda}{\lambda}} e^{-\alpha t}\|\phi\|_{\mathcal{C}^{1}}, \quad \forall t \geq 0,
$$

which implies that the closed-loop system is $\alpha$-stable. This completes our proof.

Next, we provide a numerical example to show the effectiveness of our result.

Example 1. Consider the system (1), where

$$
\begin{aligned}
A_{0} & =\left[\begin{array}{cc}
-3 & 0 \\
0.5 & -0.9
\end{array}\right], & A_{1} & =\left[\begin{array}{cc}
0.1 & 0 \\
-0.5 & 0.3
\end{array}\right], \\
B & =\left[\begin{array}{l}
1 \\
3
\end{array}\right], & C & =\left[\begin{array}{ll}
1 & 0.1
\end{array}\right]
\end{aligned}
$$

with

$$
\left\{\begin{aligned}
h(t)= & 0.1+0.8 \sin ^{2} t \\
& \text { if } \quad t \in \mathcal{I}=\cup_{k \geq 0}[2 k \pi,(2 k+1) \pi], \\
h(t)= & 0.1 \quad \text { if } \quad t \in \mathbb{R}^{+} \backslash \mathcal{I},
\end{aligned}\right.
$$

and $\phi(t)=\left[\begin{array}{ll}\cos t & \sin t\end{array}\right]^{T}$. 
It is worth noticing that the delay function $h(t)$ is non-differentiable, and therefore the design methods using observer-based and dynamic output feedback controllers for time-delay systems in the existing papers (Baser and Kizilsac, 2007; Chen, 2007; Ivanescu et al., 2000; Kwon et al., 2006; Park, 2004) are not applicable to this example.

We have that $\alpha=0.4$. By using the LMI Toolbox from MATLAB, the LMIs in Theorem 1 are satisfied with $h_{1}=0.1, h_{2}=0.9$, and

$$
\begin{aligned}
P & =\left[\begin{array}{cc}
0.8186 & -0.1397 \\
-0.1397 & 8.9918
\end{array}\right], \\
Q_{1} & =\left[\begin{array}{cc}
0.1698 & -0.0874 \\
-0.0874 & 2.3185
\end{array}\right], \\
Q_{2} & =\left[\begin{array}{cc}
0.2237 & -0.4782 \\
-0.4782 & 2.6813
\end{array}\right], \\
S_{1} & =\left[\begin{array}{cc}
2.9162 & -0.5388 \\
-0.5388 & 3.9691
\end{array}\right], \\
S_{2} & =\left[\begin{array}{cc}
0.2129 & -0.4144 \\
-0.4144 & 6.4924
\end{array}\right], \\
M_{1} & =\left[\begin{array}{cc}
-0.1300 & 0.0907 \\
0.0244 & -0.7705
\end{array}\right], \\
M_{2} & =\left[\begin{array}{cc}
0.1550 & -0.1804 \\
-0.1080 & 2.0655
\end{array}\right], \\
N_{1} & =\left[\begin{array}{cc}
0.0270 & -0.0994 \\
-0.0210 & -0.0484
\end{array}\right], \\
N_{2} & =\left[\begin{array}{cc}
-0.0839 & 0.1947 \\
0.1989 & -1.1023
\end{array}\right], \\
Y & =\left[\begin{array}{ll}
-0.3274 & 1.3559
\end{array}\right] .
\end{aligned}
$$

Therefore, the resulting controller and observer gains for the system (1) are

$$
\begin{aligned}
& K=Y P^{-1}=\left[\begin{array}{ll}
-0.3752 & 0.1450
\end{array}\right], \\
& L=-\frac{1}{2} P C^{T}=\left[\begin{array}{l}
-0.4023 \\
-0.3797
\end{array}\right] .
\end{aligned}
$$

This implies that the system (1) is 0.4 -stable by the observer-based controller with controller and observer gains given in (20). Moreover, the solution of the closed-loop system satisfies

$$
\|x(t, \phi)\| \leq 5.1005 e^{-0.4 t}\|\phi\|_{C^{1}} .
$$

Figure 1 shows the closed-loop trajectories of the state variables $x_{1}(t)$ and $x_{2}(t)$. The simulation shows that the state trajectories converge to zeros.

Further simulation studies have also been conducted for cases where the interval time-varying $h(t)$ is non-differentiable and varies in the interval $[0.1,0.9]$. In all the cases studied, it was found that the trajectories of the state variables $x_{1}(t)$ and $x_{2}(t)$ all exponentially converged to zeros.

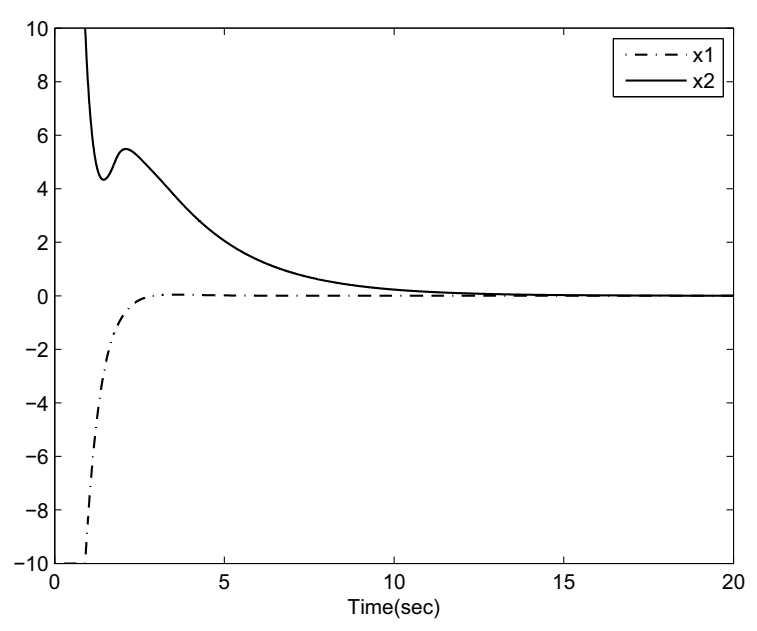

Fig. 1. Closed-loop trajectories of $x_{1}(t)$ and $x_{2}(t)$.

\section{Conclusion}

This paper has studied the problem of designing observer-based output feedback controllers for linear systems with an interval non-differentiable time-varying delay in the state vector. The design can be carried out by an efficient LMI-based algorithm. A numerical example has been given to demonstrate the simplicity of the design procedure.

\section{Acknowledgment}

This work is partly supported by the Faculty Strategic Fund, Deakin University, and the National Foundation for Science and Technology Development, Vietnam, under the grant no. 101.01.2011.51.

\section{References}

Baser, U. and Kizilsac, B. (2007). Dynamic output feedback $H_{\infty}$ control problem for linear neutral systems, IEEE Transactions on Automatic Control 52(6): 1113-1118.

Blizorukova, M., Kappel, F. and Maksimov, V. (2001). A problem of robust control of a system with time delay, International Journal of Applied Mathematics and Computer Science 11(4): 821-834.

Botmart, T., Niamsup, P. and Phat, V.N. (2011). Delay-dependent exponential stabilization for uncertain linear systems with interval non-differentiable time-varying delays, Applied Mathematics and Computation 217(21): 8236-8247.

Busłowicz, M. (2010). Robust stability of positive continuous-time linear systems with delays, International Journal of Applied Mathematics and Computer Science 20(4): 665-670, DOI: 10.2478/v10006-010-0049-8. 
Chen, J.D. (2007). Robust $\mathrm{H}_{\infty}$ output dynamic observer-based control design of uncertain neutral systems, Journal of $\mathrm{Op}$ timization Theory and Applications 132(1): 193-211.

Fridman, E. and Shaked, U. (2002). A descriptor system approach to $\mathrm{H}_{\infty}$ control of linear time-delay systems, IEEE Transactions on Automatic Control 47(2): 253-270.

Gu, K., Kharitonov, V. L. and Chen, J. (2003). Stability of TimeDelay Systems, Birkhauser, Boston, MA.

Ivanescu, D., Dion, J.M., Dugard, L. and Niculescu, S.I. (2000). Dynamical compensation for time-delay systems: An LMI approach, International Journal of Robust and Nonlinear Control 10(8): 611-628.

Kaczorek, T. and Busłowicz, M. (2004). Minimal realization for positive multivariable linear systems with delay, International Journal of Applied Mathematics and Computer Science 14(2): 181-187.

Kowalewski, A. (2009). Time-optimal control of infinite order hyperbolic systems with time delays, International Journal of Applied Mathematics and Computer Science 19(4): 597-608, DOI: 10.2478/v10006-009-0047-x.

Kwon, O.M., Park, J.H., Lee, S.M. and Won, S.C. (2006). LMI optimization approach to observer-based controller design of uncertain time-delay systems via delayed feedback, Journal of Optimization Theory and Applications 128(1): 103-117.

Park, J.H. (2004). On the design of observer-based controller of linear neutral delay-differential systems, Applied Mathematics and Computation 150(1): 195-202.

Park, P. (1999). A delay-dependent stability criterion for systems with uncertain time-invariant delays, IEEE Transactions on Automatic Control 44(4): 876-877.

Phat, V.N., Khongtham, Y. and Ratchagit, K. (2012). LMI approach to exponential stability of linear systems with interval time-varying delays, Linear Algebra and Its Applications 436(1): 243-251.

Raja, R., Sakthivel, R., Anthoni, S.M. and Kim, H. (2011). Stability of impulsive Hopfield neural networks with Markovian switching and time-varying delays, International Journal of Applied Mathematics and Computer Science 21(1): 127-135, DOI: 10.2478/v10006-011-0009-y.

Richard, J.P. (2003). Time-delay systems: An overview of some recent advances and open problems, Automatica 39(10): 1667-1694.

Shao, H. (2009). New delay-dependent stability criteria for systems with interval delay, Automatica 45(3): 744-749.

Shao, H. and Han, Q.L. (2012). Less conservative delay-dependent stability criteria for linear systems with interval time-varying delays, International Journal of Systems Science 43(5): 894-902.

Tokarzewski, J. (2009). Zeros in linear systems with time delay in state, International Journal of Applied Mathematics and Computer Science 19(4): 609-617, DOI: 10.2478/v10006-009-0048-9.
Tong, S., Yang, G. and Zhang, W. (2011). Observer-based fault-tolerant control against sensor failures for fuzzy systems with time delays, International Journal of Applied Mathematics and Computer Science 21(4): 617-627, DOI: 10.2478/v10006-011-0048-4.

Trinh, H. (1999). Linear functional state observer for time-delay systems, International Journal of Control 72(18): 1642-1658.

Trinh, H. and Aldeen, M. (1994). Stabilization of uncertain dynamic delay systems by memoryless feedback controllers, International Journal of Control 59(6): 1525-1542.

Trinh, H. and Aldeen, M. (1997). On robustness and stabilization of linear systems with delayed nonlinear perturbations, IEEE Transactions on Automatic Control 42(7): 1005-1007.

Trinh, H.M., Teh, P.S. and Fernando, T.L. (2010) Time-delay systems: Design of delay-free and low-order observers, IEEE Transactions on Automatic Control 55(10): 2434-2438.

Xiang, Z., Wang, R. and Chen, Q. (2010). Fault tolerant control of switched nonlinear systems with time delay under asynchronous switching, International Journal of Applied Mathematics and Computer Science 20(3): 497-506, DOI: 10.2478/v10006-010-0036-0.

Mai Viet Thuan received the M.Sc. degree in mathematics at the Institute of Mathematics, Vietnam Academy of Science and Technology, Hanoi, Vietnam, in 2009. Since 2007, he has been a lecturer at Thai Nguyen University, Vietnam. He is currently a Ph.D. research student at the Institute of Mathematics, Vietnam Academy of Science and Technology. His research interests include stability and stabilization of timevarying delay systems.

Vu Ngoc Phat received the B.Sc. and Ph.D. degrees in mathematics at the former USSR Bacu State University in 1975 and 1984, respectively. In 1995, he received the D.Sc. degree in mathematics at the Institute of Mathematics, Polish Academy of Sciences, Poland. He was a senior research fellow at Pusan National University, South Korea (19981999) and the University of New South Wales, Australia (2001-2003). Currently, he works as a professor at the Institute of Mathematics, Vietnam Academy of Science and Technology, Hanoi, Vietnam. He is the author/co-author of two monographs and 95 refereed journal papers. His research interests include systems and control theories, optimization techniques, stability analysis, and time-delay systems.

Hieu Trinh received the B.Eng. (Hons.), M.Eng.Sc. and Ph.D. degrees in electrical and electronic engineering from the University of Melbourne, Australia, in 1990, 1992 and 1996, respectively. Between March 1995 and December 1996, he was a postdoctoral research fellow at the Department of Electrical and Electronic Engineering, University of Melbourne. Between January 1997 and December 2000, he was a lecturer at James Cook University, Townsville, Australia. He joined Deakin University, Australia, in January 2001, where he is currently an associate professor. His present research activities are in the areas of systems and control theories, robotics, power systems and application of control theory to industrial systems. Professor Trinh is the author/co-author of about 70 refereed journal papers and a research monograph entitled Functional Observers for Dynamical Systems (Springer).

Received: 4 January 2012

Revised: 20 May 2012 